\title{
The effect of center volume on the incidence of postoperative complications and their impact on survival after lung transplantation
}

\author{
Arman Kilic, MD, ${ }^{\text {a }}$ Timothy J. George, MD, ${ }^{\text {a }}$ Claude A. Beaty, MD, ${ }^{\text {a Christian A. Merlo, MD, MPH, }}$ \\ John V. Conte, MD, ${ }^{a}$ and Ashish S. Shah, MD $^{\mathrm{a}}$
}

Objective: The aim of this study was to evaluate the effect of center volume on the incidence of postoperative complications and their impact on survival after lung transplantation (LTx).

\begin{abstract}
Methods: United Network for Organ Sharing data were used to identify adult patients undergoing LTx between 1999 and 2009. Center volume was modeled as both a continuous and a categorical variable. Postoperative complications included infection, rejection, stroke, reoperation, and renal failure requiring dialysis. Multivariable Cox regression and Kaplan-Meier analyses were conducted after stratification on the basis of center volume and type of complication.
\end{abstract}

Results: A total of 12,565 LTx recipients were included in the study. Overall rates of postoperative complications were $5.4 \%$ for renal failure requiring dialysis, $1.9 \%$ for stroke, $19.9 \%$ for reoperation, $42.8 \%$ for infection, and $10.0 \%$ for rejection. High volume centers did not have significantly reduced rates of postoperative complications. Risk-adjusted multivariable Cox analysis demonstrated that in patients with a complication, low volume center was a significant risk factor for increased 90-day, 1-year, and 5-year mortality. KaplanMeier analyses similarly demonstrated reduced posttransplant survival in lower volume centers, a finding that persisted after stratification based on individual complication type except for stroke.

Conclusions: Although high volume centers do not have significantly lower incidences of individual postoperative complications after LTx, they are best able to minimize the adverse effects of these complications on shortand long-term survival. These data suggest that identifying and implementing the institutional practices that lead to better management of postoperative complications after LTx in high volume centers may be prudent to improving outcomes in lower volume hospitals. (J Thorac Cardiovasc Surg 2012;144:1502-9)

Prior studies have demonstrated that higher center volume is associated with improved survival after lung transplantation (LTx). ${ }^{1,2}$ However, the mechanisms by which higher volume leads to reduced mortality remain unknown. Some of these mechanisms may be related to postoperative complications. Moreover, centers that are able to reduce the rates of postoperative complications, recognize complications early in their course, and/or manage them effectively once they

From the Division of Cardiac Surgery, ${ }^{\mathrm{a}}$ Department of Surgery, and the Division of Pulmonary and Critical Care Medicine, ${ }^{\mathrm{b}}$ Department of Medicine, The Johns Hopkins Medical Institutions, Baltimore, Md.

This work was supported in part by Health Resources and Services Administration contract 234-2005-37011C. The content is the responsibility of the authors alone and does not necessarily reflect the views or policies of the Department of Health and Human Services, nor does mention of trade names, commercial products, or organizations imply endorsement by the US Government. Departmental funds were received from the Department of Surgery, Johns Hopkins Hospital, Baltimore, Md.

Disclosures: Authors have nothing to disclose with regard to commercial support.

Read at the 92nd Annual Meeting of The American Association for Thoracic Surgery, San Francisco, California, April 28-May 2, 2012.

Received for publication March 29, 2012; revisions received July 10, 2012; accepted for publication Aug 21, 2012; available ahead of print Sept 19, 2012

Address for reprints: Ashish S. Shah, MD, Division of Cardiac Surgery, The Johns Hopkins Hospital, 600 N Wolfe St, Blalock 618, Baltimore, MD 21287 (E-mail: ashah29@jhmi.edu).

$0022-5223 / \$ 36.00$

Copyright (c) 2012 by The American Association for Thoracic Surgery http://dx.doi.org/10.1016/j.jtcvs.2012.08.047 occur should theoretically have improved outcomes. It is unclear whether center volume affects these factors. Therefore, in this study, we analyzed the effect of center volume on the incidence of postoperative complications and their impact on short- and long-term survival after LTx.

\section{PATIENTS AND METHODS \\ Data Source}

Data from the United Network for Organ Sharing (UNOS) were obtained for the purposes of this analysis. The UNOS registry is a publically available deidentified data set that contains patient-level data on all transplantations performed in the United States. The Johns Hopkins University School of Medicine Institutional Review Board approved this study.

\section{Inclusion and Exclusion Criteria}

We included all adult patients over the age of 17 years who underwent LTx between January 1, 1999, and December 31, 2009. Pediatric patients were excluded from the study. Patients undergoing redo LTx, heart-lung transplantation, or other types of multivisceral transplantation were also excluded.

\section{Center Volume and Postoperative Complications}

Patients were initially distributed into equal-sized tertiles on the basis of center LTx volume. These tertiles were used to define low, intermediate, and high volume centers. The rates of postoperative complications were then compared among these cohorts. Postoperative complications were defined as occurring before discharge after LTx. The individual complications that were included in our analysis were renal failure requiring dialysis, 

Abbreviations and Acronyms
CI = confidence interval
LTX = lung transplantation
OR = odds ratio
UNOS $=$ United Network for Organ Sharing

stroke, all-cause reoperation, infection, and rejection. Risk-adjusted analysis was also performed incorporating univariate predictors $(P<.2)$ of these complications to evaluate the independent effect of center volume both as a continuous variable and as a categorical variable on these adverse event rates.

The availability of complications data within the UNOS database was different for different complication types. Moreover, renal failure requiring dialysis and stroke were available uniformly throughout our study period from 1999 to 2009 . Only $2.1 \%$ and $2.3 \%$ of data were missing for these complications, respectively. Reoperation and infection were only available from 1999 to 2006, with no coding from 2007 onward. In the years that these complications were available, data were missing in only $3.1 \%$ and $3.2 \%$, respectively. Finally, rejection occurring before discharge, although coded throughout the study period, was poorly coded before 2004 . From 2004 to 2009 , only $4.2 \%$ of data were missing. The data presented herein represent data for these specific years for each complication type.

After examining the rates of postoperative complications, we evaluated the impact of these complications on short- and long-term survival, stratified by center volume. Moreover, end points included 90-day, 1-year, and 5 -year all-cause mortality, as well as 1 -year mortality excluding 90 -day deaths and 5-year mortality excluding 1-year deaths. Multivariable Cox regression analyses incorporating recipient, donor, and transplant variables associated with these end points in univariate analysis $(P<.2$ in exploratory analysis) were conducted, as were Kaplan-Meier analyses. Statistical analyses were performed using STATA software version 11 (StataCorp LP, College Station, Tex).

\section{RESULTS}

\section{Baseline Characteristics}

A total of 12,565 adult patients underwent LTx at 77 transplant centers between 1999 and 2009 and met eligibility criteria for our study. The average annual center volume for the overall cohort was $31.1 \pm 17.9$ LTx per year. After patients were divided into equal-sized tertiles, this corresponded to the following thresholds: low $(<21.8$ LTx per year), intermediate (21.8-34.2 LTx per year), and high (>34.2 LTx per year) volume.

When the annual volume of LTx at each individual center across the study period were examined, there were $9(11.7 \%)$ programs that "came online," meaning they did not initially perform any transplants in the earlier part of the study period and then began performing LTx in later years. Each of these programs was categorized as low volume based on their aggregate volume, and when examined on an annual basis, all but $2(2.6 \%)$ had indeed been low volume for each of the years in the study period. These other 2 programs were initially low volume but had consistently grown over the study period and were in fact high volume by the end of the study interval. Excluding these centers produced the same results; therefore, for simplicity, we included these 2 centers in the low volume cohort based on their aggregate volume. Overall, the standard deviation within each individual center for annual LTx volume was 6.2 transplants.

There were several differences when comparing key baseline recipient characteristics between center volume cohorts (Table 1). High volume centers tended to transplant older patients, more male patients, and more white patients. Serum creatinine was statistically higher in low volume centers. There were also differences in etiology of lung disease, such that high volume centers had a higher proportion of idiopathic pulmonary fibrosis as compared with low volume centers. Finally, there was a higher acuity of recipients at high volume centers, inasmuch as the proportion of patients bridged with mechanical ventilation or extracorporeal membrane oxygenation was significantly higher at these institutions.

There were key differences in donor characteristics as well (Table 1). High volume centers used older donors and also had a higher frequency of female donors. A higher proportion of donors at high volume centers were white, and fewer had a traumatic mechanism of death. With respect to transplant variables, high volume centers used bilateral LTx more frequently than low or intermediate volume centers (Table 1). High volume centers also had longer ischemic times, which was in part a reflection of procuring organs from donors located at greater distances from the transplant center.

\section{Rates of Postoperative Complications}

The overall rates of complications for the entire study cohort were as follows: $5.4 \%$ for renal failure requiring dialysis, $1.9 \%$ for stroke, $19.9 \%$ for reoperation, $42.8 \%$ for infection, and $10.0 \%$ for rejection. In examining temporal trends, the rates of renal failure, stroke, and reoperation did not significantly change over time. Conversely, the rate of infection significantly increased from $40.9 \%$ in 1999 to $46.9 \%$ in 2006 ( $P$ value for trend $=.008)$. The rate of rejection significantly declined from $13.2 \%$ in 2004 to $9.0 \%$ in 2009 ( $P$ value for trend $=.002$ ).

There were significant differences in the rates of postoperative complications by center volume cohort, except for stroke, which was comparable between groups (Figure 1). More specifically, intermediate and high volume centers had a comparable rate of renal failure requiring dialysis ( $4.9 \%$ and $4.7 \%$, respectively), which was significantly lower than the rate of $6.5 \%$ observed in low volume centers $(P<.001)$. For the remaining 3 complications (reoperation, infection, and rejection), intermediate volume centers consistently had the lowest rate, which was significantly less than the rates observed in low and high volume centers (each $P<.05$ ) (Figure 1).

After risk adjustment, center volume displayed comparable trends to unadjusted analysis when modeled as a categorical variable. Moreover, low volume centers had significantly higher odds of postoperative dialysis (odds 
TABLE 1. Baseline characteristics stratified by center volume

\begin{tabular}{|c|c|c|c|c|}
\hline Variable & Low volume $(n=4460)$ & Intermediate volume $(n=4227)$ & High volume $(\mathrm{n}=3878)$ & $P$ value* \\
\hline \multicolumn{5}{|l|}{ Recipient } \\
\hline Age (y) & $51.4 \pm 13.0$ & $52.3 \pm 12.4$ & $53.1 \pm 12.5$ & $<.001$ \\
\hline Female gender & $2086(46.8 \%)$ & $1939(45.9 \%)$ & $1688(43.5 \%)$ & .01 \\
\hline White race & $3723 / 4440(83.9 \%)$ & $3670 / 4218(87.0 \%)$ & $3484 / 3871(90.0 \%)$ & $<.001$ \\
\hline Diagnosis & & & & .03 \\
\hline COPD & $1663(37.3 \%)$ & $1520(36.0 \%)$ & $1355(34.9 \%)$ & \\
\hline IPF & $1269(28.5 \%)$ & $1283(30.4 \%)$ & $1190(30.7 \%)$ & \\
\hline $\mathrm{CF}$ & $649(14.6 \%)$ & $574(13.6 \%)$ & $504(13.0 \%)$ & \\
\hline Other & $879(19.7 \%)$ & $850(20.1 \%)$ & $829(21.4 \%)$ & \\
\hline Bridging with ECMO & $18(0.4 \%)$ & $20(0.5 \%)$ & $38(1.0 \%)$ & .001 \\
\hline Mechanical ventilation & $120(2.7 \%)$ & $160(3.8 \%)$ & $166(4.3 \%)$ & $<.001$ \\
\hline Serum creatinine $(\mathrm{mg} / \mathrm{dL})$ & $0.91 \pm 0.60$ & $0.88 \pm 0.44$ & $0.88 \pm 0.56$ & .01 \\
\hline \multicolumn{5}{|l|}{ Donor } \\
\hline Age (y) & $32.4 \pm 13.8$ & $32.4 \pm 13.8$ & $34.3 \pm 14.7$ & $<.001$ \\
\hline Female gender & $1707(38.3 \%)$ & $1670(39.5 \%)$ & $1623(41.9 \%)$ & .004 \\
\hline White race & $2881 / 4441(64.9 \%)$ & $2824 / 4212(67.1 \%)$ & $2789 / 3863(72.2 \%)$ & $<.001$ \\
\hline Trauma as mechanism of death & $2297 / 4437(51.8 \%)$ & $2155 / 4186(51.5 \%)$ & $1765 / 3869(45.6 \%)$ & $<.001$ \\
\hline \multicolumn{5}{|l|}{ Transplant } \\
\hline Bilateral LTx & $2254(50.5 \%)$ & $2065(48.9 \%)$ & $2728(70.4 \%)$ & $<.001$ \\
\hline $\begin{array}{l}\text { Median distance between donor } \\
\text { hospital and transplant center (mile) }\end{array}$ & 68.9 (IQR 7.4-210.0) & 97.8 (IQR 15.5-269.1) & 196.2 (IQR 49.5-359.3) & $<.001$ \\
\hline Ischemic time (h) & $4.5 \pm 1.6$ & $4.8 \pm 1.7$ & $5.4 \pm 1.7$ & $<.001$ \\
\hline
\end{tabular}

Continuous data were presented as mean \pm standard deviation, except for distance between donor hospital and transplant center, which was presented as median with interquartile range. Frequency data were presented as number (percentage). All percentages for frequency data were based on all patients within the cohort unless otherwise indicated. $C O P D$, Chronic obstructive pulmonary disease; $I P F$, interstitial pulmonary fibrosis; $C F$, cystic fibrosis; $E C M O$, extracorporeal membrane oxygenation; $L T x$, lung transplantation; $I Q R$, interquartile ratio. ${ }^{*} P$ value based on $\chi^{2}$ for frequency data and analysis of variance for continuous data. $P$ value for median distance based on Kruskal-Wallis nonparametric test.

ratio (OR), $1.79 ; P<.001)$, reoperation $(\mathrm{OR}, 1.28$; $P=.007)$, and infection (OR, $1.27 ; P=.001)$, and significantly lower odds of rejection (OR, $0.79 ; P=.02)$ as compared with high volume centers. The risk-adjusted odds of stroke were comparable in low $(\mathrm{OR}, 1.24 ; P=.25)$ versus high volume centers. When compared with high volume centers, intermediate volume centers had comparable riskadjusted odds of stroke (OR, 1.22, $P=.28$ ) and reoperation (OR, 0.90; $P=.26$ ), higher risk-adjusted odds of renal failure (OR, $1.31 ; P=.04$ ), and significantly lower riskadjusted odds of infection (OR, $0.78 ; P=.001)$ and rejection (OR, $0.55 ; P<.001)$.

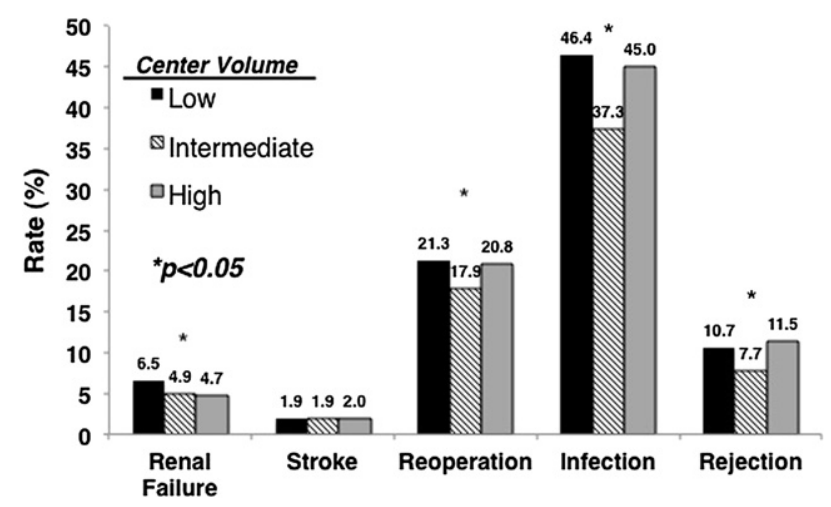

FIGURE 1. Rates of postoperative complications by center volume.
When modeled as a continuous variable, decreasing center volume was found to be associated with a significantly increased risk-adjusted likelihood of renal failure (OR, $1.01 ; P<.001)$ and infection $(\mathrm{OR}, 1.01 ; P<.001)$. The risk-adjusted odds of stroke, reoperation, and rejection were not significantly affected by center volume as a continuous variable (each $P>.05$ ).

\section{Impact of Complications on Survival}

Each individual complication significantly increased the likelihood of risk-adjusted posttransplant mortality (each $P \leq .001$ ) when examined independent of each other. The hazard ratio for death ranged from 1.26 in those with rejection to 4.18 in those with renal failure requiring dialysis after LTx. Kaplan-Meier analysis excluding patients with multiple complications demonstrated that 5 -year survivals ranged from $61.0 \%$ (95\% confidence interval [CI], $58.1 \%-63.7 \%$ ) in those with no complications to $15.4 \%$ (95\% CI, $1.2 \%-45.3 \%)$ in those with postoperative renal failure requiring dialysis $(\log -$ rank $P$ value $<.001)$. Shorter-term survival displayed the same trends, with 90day and 1-year survivals ranging from $97.2 \%$ (95\% CI, 96.2\%-97.9\%) and $91.1 \%$ (95\% CI, 89.5\%-92.5\%) in those with no complications to $53.9 \%$ (95\% CI, $24.8 \%-76.0 \%$ ) and $46.2 \%$ (95\% CI, 19.2\%-69.6\%) in patients requiring dialysis, respectively. 
Similarly, Kaplan-Meier analysis confirmed that an increasing number of complications was associated with significantly decreasing short- and long-term survivals (Figure 2). Moreover, 90-day survival ranged from 97.2\% (95\% CI, 96.2\%-97.9\%) in patients with no complications to $67.3 \%(95 \% \mathrm{CI}, 60.5 \%-73.2 \%)$ in patients with at least 3 complications $(P<.001)$. Survival at 1 year ranged from $91.1 \%$ (95\% CI, $89.5 \%-92.5 \%$ ) to $45.3 \%$ (95\% CI, $38.5 \%$ $51.9 \%$ ) and at 5 years from $61.0 \%$ (95\% CI, 58.1\%$63.7 \%$ ) to $27.6 \%$ (95\% CI, $21.6 \%-33.8 \%$ ) in patients with no complications versus those with 3 or more complications, respectively (each $P<.001$ ). In risk-adjusted analysis incorporating all complications, renal failure $(\mathrm{OR}, 3.84 ; P<$ $.001)$, stroke (OR, 1.66; $P=.008$ ), and reoperation (OR, $1.71 ; P<.001)$ were all associated with an independent increase in odds of post-LTx mortality, whereas the adverse effect of early rejection (OR, $1.09 ; P=.36)$ and infection (OR, $1.01 ; P=.84)$ no longer persisted.

\section{Impact of Center Volume on Survival in Patients With Complications}

In risk-adjusted multivariable Cox regression models limited to patients with a postoperative complication, center volume was found to significantly affect post-LTx mortality (Table 2). More specifically, when modeled both as a continuous and categorical variable, decreasing center volume correlated with a significant increase in 90-day, 1-year, and 5-year mortality risk. The strongest effect was at the 90-day mark, where recipients undergoing LTx with a postoperative complication at a low volume center were at a $52 \%$ increased risk of mortality compared with patients experiencing a complication at a high volume center $(P<.001)$. Low volume centers similarly exhibited a $46 \%$ and $32 \%$ increased risk of mortality relative to high volume centers at 1 year and 5 years in these cases with complications (each $P<.001)$. To ensure that the effects of center volume on overall 1- and 5-year mortality were not accounted for by the effects on earlier mortality, we conducted a time-segmented analysis. Moreover, in this analysis, center volume both as

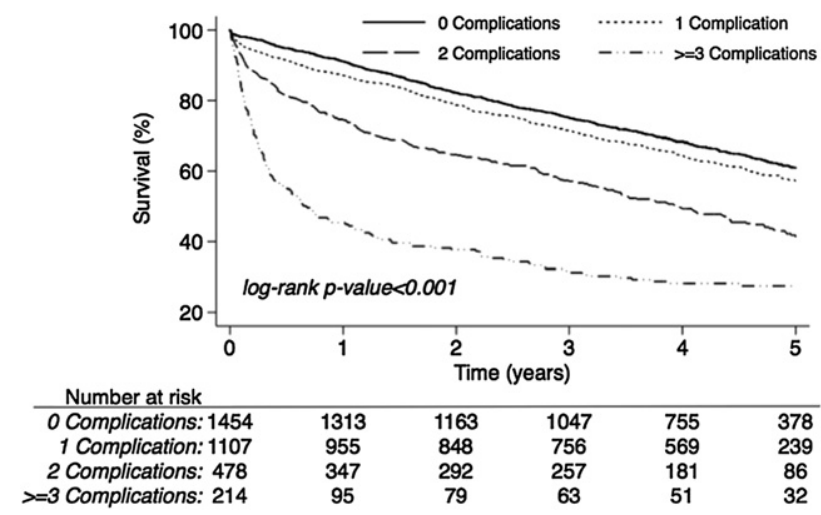

FIGURE 2. Kaplan-Meier survival stratified by number of complications. a continuous and categorical variable was found to affect 1 -year mortality excluding 90-day mortalities, as well as 5 -year mortality excluding 1-year mortalities (Table 3 ).

Kaplan-Meier analysis confirmed these findings. Moreover, high volume centers had improved 90 -day $(88.8 \%$ vs $84.2 \% ; P<.001), 1$-year $(77.9 \%$ vs $71.8 \% ; P<.001)$, and 5 -year $(49.1 \%$ vs $38.9 \% ; P<.001)$ survivals as compared with low volume centers in patients with a postoperative complication (Figure 3,A). Except for stroke, the correlation between center volume and posttransplant survival remained significant when performing separate analyses for each individual complication type (each log-rank $P$ value $<.05$ ) (Figure $3, B-F$ ).

\section{DISCUSSION}

Procedural volume has been shown to correlate with operative outcomes in numerous studies. In their landmark study, Birkmeyer and colleagues ${ }^{3}$ surveyed the Nationwide Inpatient Sample and Medicare claims database to examine the impact of hospital volume on operative mortality in 6 different cardiovascular procedures and 8 major cancer resections. ${ }^{3}$ They found in their analysis of over 2.5 million procedures that higher volume hospitals had reduced risk-adjusted operative mortality for all procedures. This study along with others helped form the foundation for the Leapfrog recommendations and other evidence-based hospital referrals. ${ }^{4,5}$

In the realm of LTx, several studies have shown similar results. Weiss and coworkers ${ }^{1}$ examined over 10,000 primary LTx cases from the UNOS database and found that recipients at low volume centers were at increased risk of short-term mortality. Scarborough and coworkers ${ }^{2}$ also demonstrated the significant association between center volume and 1-year mortality in LTx and, in addition, found that the volume-outcomes relationship has become even more pronounced over time.

A central question that has arisen from these studies is what drives this relationship between procedural volume and mortality. One theoretical contributor may be related to postoperative complications. Moreover, it can be postulated that high volume centers achieve improved survival by reducing the rates of postoperative complications, recognizing their occurrence earlier, and/or better managing them once they occur.

Therefore, in this study, our aim was to evaluate the effect of center volume on the incidence of complications and their impact on survival after LTx. The principal finding in our analysis of over 12,000 LTx cases was that although high volume centers do not have significantly lower incidences of postoperative complications, they are best able to mitigate the adverse effects of these complications on short- and long-term survival.

\section{Prior Studies}

Several prior studies have similarly examined the effect of center volume on rates of complications and their impact 
TABLE 2. Risk-adjusted impact of center volume on 90-day, 1-year, and 5-year post-LTx mortality in patients with a postoperative complication

\begin{tabular}{|c|c|c|c|}
\hline Center volume & $\frac{\text { Ninety-day }}{\text { HR }(95 \% \mathrm{CI})}$ & $\frac{\text { One-year }}{\text { HR }(95 \% \text { CI })}$ & $\frac{\text { Five-year }}{\text { HR }(95 \% \mathrm{CI})}$ \\
\hline Decreasing center volume (continuous) & $1.01(1.01-1.02) \S$ & $1.01(1.00-1.01) \S$ & $1.01(1.00-1.01) \S$ \\
\hline \multicolumn{4}{|l|}{ Center volume (categorical) } \\
\hline High & Reference & Reference & Reference \\
\hline Intermediate & $1.30(1.30-1.64) \S$ & $1.18(0.99-1.41)$ & $1.03(0.90-1.18)$ \\
\hline Low & $1.52(1.22-1.90) \S$ & $1.46(1.24-1.72) \S$ & $1.32(1.17-1.50) \S$ \\
\hline
\end{tabular}

$H R$, Hazard ratio; $C I$, confidence interval. *Risk-adjusted for univariate predictors $(P<.2)$ of 90 -day mortality: age, weight, serum creatinine, serum bilirubin, body mass index, race, gender, mechanical ventilation, intensive care unit before transplant, etiology of lung disease, dialysis while on wait list, red blood cell transfusion while on wait list, donor weight, donor cytomegalovirus status, donor race, donor/recipient body mass index ratio, recipient-donor race-matching, and bridge to transplant with extracorporeal membrane oxygenation. $\dagger$ Risk-adjusted for univariate predictors $(P<.2)$ of 1-year mortality: age, weight, single versus bilateral lung transplantation, serum creatinine, serum bilirubin, body mass index, race, gender, mechanical ventilation, intensive care unit before transplant, etiology of lung disease, dialysis while on wait list, red blood cell transfusion while on wait list, donor weight, donor cytomegalovirus status, donor diabetes, donor terminal creatinine, donor race, donor/recipient body mass index ratio, recipient/donor race-matching, and bridge to transplant with extracorporeal membrane oxygenation. ŁRisk-adjusted for univariate predictors $(P<.2)$ of 5 -year mortality: age, weight, single versus bilateral lung transplantation, serum creatinine, serum bilirubin, days on wait list, body mass index, gender, mechanical ventilation, intensive care unit before transplant, etiology of lung disease, recipient cytomegalovirus status, dialysis while on wait list, red blood cell transfusion while on wait list, donor age, donor cytomegalovirus status, donor cigarette use, donor diabetes, donor race, donor/recipient body mass index ratio, ischemic time, and bridge to transplant with extracorporeal membrane oxygenation. $\S P<.05$.

on surgical mortality. A study of over 35,000 pediatric heart surgery patients from the Society of Thoracic Surgeons Congenital Heart Surgery Database demonstrated no association between center volume and rates of complications, but it did show a significant association between center volume and mortality from complications, similar to our analysis. $^{6}$ Another study by Ghaferi, Birkmeyer, and Dimick ${ }^{7}$ used Medicare data to examine this issue in patients undergoing gastrectomy, pancreatectomy, and esophagectomy. They found that differences in mortality between low and high volume centers were not attributable to significant differences in complication rates but rather the ability of hospitals to "rescue" patients from these complications, or in other words, prevent mortality once the complication occurred.

Another study of 1864 patients undergoing gastrectomy for gastric cancer at 214 hospitals located in Texas similarly demonstrated that although adverse event rates were comparable between low and high volume hospitals, mortality rates in patients with these events were significantly increased at low volume centers. ${ }^{8}$ These studies support the notion that the ability to recognize and effectively manage major postoperative complications may be a mechanism by which higher volume institutions achieve improved outcomes.

\section{Potential Reasons for Our Findings}

The reasons why higher volume centers have improved survival after LTx in patients with a postoperative complication despite similar rates of occurrence are difficult to ascertain from variables available in the UNOS dataset. One potential reason may be related to nursing and intensive care unit staffing. In the aforementioned study of gastrectomies by Smith and colleagues, ${ }^{8}$ for instance, fewer critical care beds and higher licensed vocational nurse/patient ratios were associated with worse outcomes. The authors postulated that critical care beds may be a surrogate for the availability of expertise within the hospital, such as interventional radiology, as well as the overall experience of the center. Inasmuch as vocational nurses practice under the supervision of registered nurses, the authors also suggested that having a larger proportion of the nursing staff

TABLE 3. Risk-adjusted impact of center volume on 1-year and 5-year post-LTx mortality excluding 90-day and 1-year deaths, respectively, in patients with a postoperative complication

\begin{tabular}{lcc}
\hline \multicolumn{1}{c}{ Center volume } & One year excluding 90-day mortalities* & Five years excluding 1-year mortalities $\dagger$ \\
HR (95\% CI)
\end{tabular}

HR, Hazard ratio; $C I$, confidence interval. *Risk-adjusted for univariate predictors $(P<.2)$ of 1-year mortality excluding 90 -day mortalities: age, single versus bilateral lung transplantation, serum creatinine, year of transplant, gender, mechanical ventilation, diabetes mellitus, intensive care unit before transplant, etiology of lung disease, previous malignancy, cytomegalovirus status, red blood cell transfusion while on wait list, donor age, donor weight, donor height, donor cytomegalovirus status, donor cigarette use, donor diabetes, donor terminal creatinine, donor race, recipient-donor gender matching, recipient-donor race matching, and recipient-donor cytomegalovirus status matching. $\dagger$ Riskadjusted for univariate predictors $(P<.2)$ of 5-year mortality excluding 1-year mortalities: age, single versus bilateral lung transplantation, serum creatinine, year of transplant, days on wait list, diabetes mellitus, intensive care unit before transplant, etiology of lung disease, dialysis while on wait list, donor age, donor cytomegalovirus status, donor diabetes, donor terminal creatinine, donor race, recipient-donor race matching, and ischemic time. $\ddagger P<.05$. 

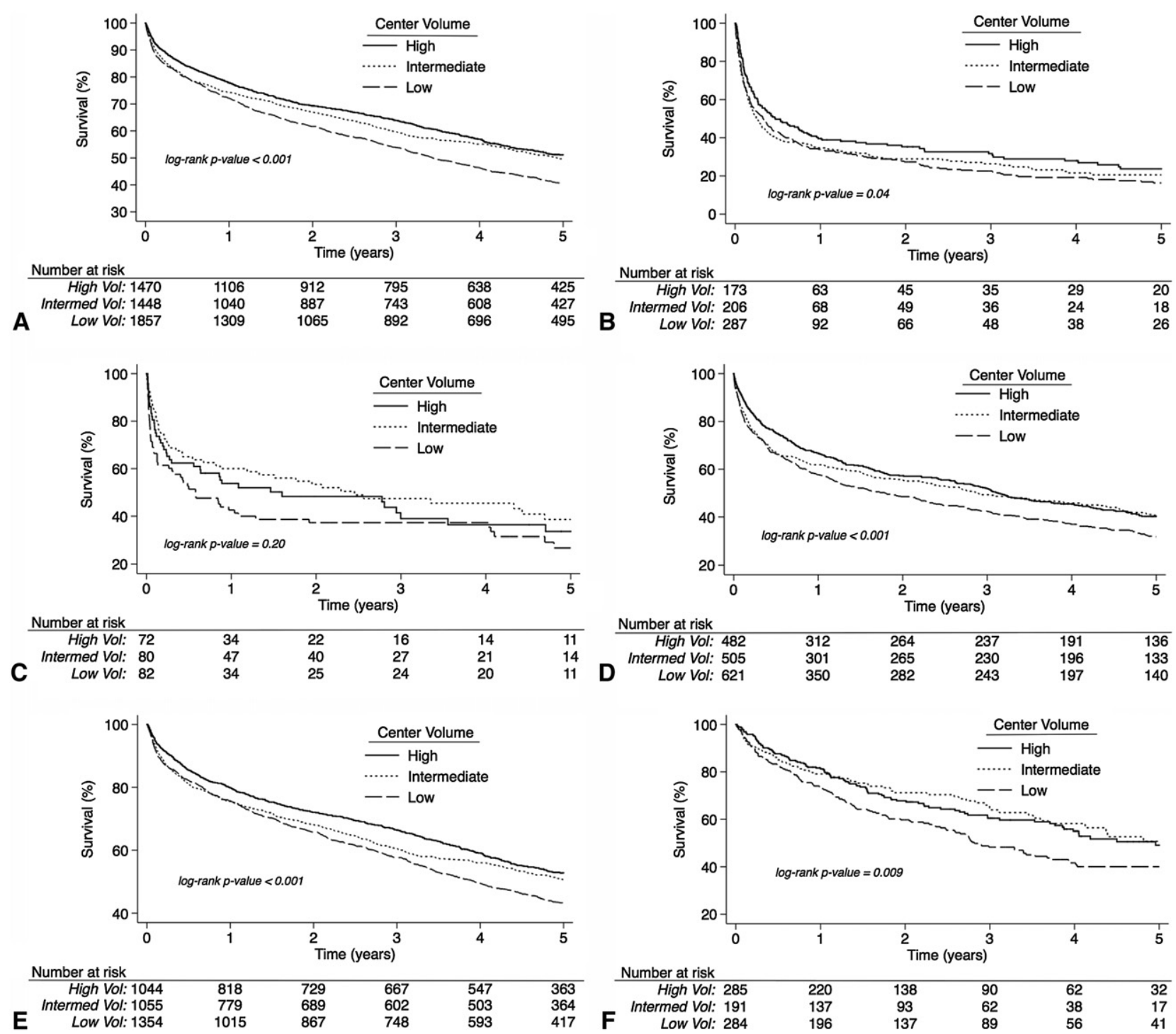

FIGURE 3. Kaplan-Meier survival curves stratified by center volume for patients with any complication (A), renal failure requiring dialysis (B), stroke (C), reoperation (D), infection (E), and rejection (F).

be comprised of vocational nurses may lead to worse outcomes inasmuch as more experienced staff may be better able to identify complications early in their course.

Other studies have confirmed this latter concept as well. Aiken and associates ${ }^{9}$ demonstrated lower surgical mortality and higher rescue from complication rates in hospitals with higher registered nurse/patient ratios. Another study by the same group found that having nurses with higher education levels similarly resulted in reduced mortality and improved rescue from complications. ${ }^{10}$

Another factor that may contribute to the findings in our study is the use of standardized clinical pathways. These pathways are more likely to be present in higher volume centers and may be important in the early recognition and effective clinical management of major complications. Although the impact of these pathways on rescue from complications or in LTx in general has not been well studied, prior studies have demonstrated their association with improved outcomes and reduced costs in other procedures. ${ }^{11-13}$

\section{Limitations}

As mentioned previously, the major limitation to our study is the absence of certain institutional variables in the UNOS database that are important to better understanding the relationship between center volume and the ability to reduce mortality from complications after LTx. These variables include nurse/patient ratios, staffing and resources in the intensive care unit, house staff coverage, standardized clinical pathways, and the availability and expertise of subspecialty services. Another limitation is that complications are simply coded as being present or absent in the UNOS registry. The degree or severity of these complications is 
not provided, although this would certainly be expected to affect mortality rates. Also, the timing of the complication in relation to the LTx is also not available in the dataset; therefore, time zero could not be defined as the time of the complication but rather had to be defined as the date of the LTx.

A limitation of our statistical analysis is that center volume is a dynamic variable that changes on an annual basis. In other words, a center that may be low volume in earlier years may become higher volume over time, or vice versa. We chose to use aggregate volume to account for these year-to-year changes. Despite this limitation, we found that the average standard deviation for annual volume within each individual center was only $6.2 \mathrm{LTx}$; furthermore, only $2(2.6 \%)$ programs that had come online during the study period would have been reclassified as their volume changed significantly over the study interval.

Additionally, aside from renal failure and stroke, complications were not uniformly coded throughout the study period for each complication type. However, in the years that the complications were available, the coding was relatively complete for the majority of patients. Furthermore, inasmuch as there is variable length of hospital stay after LTx, the "exposure" to developing early complications is also variable between patients and between centers. We also did not include quality of life data, although this is certainly an important outcome in LTx and likely correlates with rates of complications. A final limitation was that we could not determine whether patient mortality was related to a specific complication or not, and if so, to what degree. Although coding is available for the cause of death in patients in the UNOS dataset, the sequence of clinical events leading to a patient's death can be complex and the etiology of death is often multifactorial. Therefore, a simple code for 1 cause of death is often not convincing of whether the death was in actuality related in any way to a specific complication.

\section{CONCLUSIONS}

In this review of over 12,000 patients with primary LTx, we found that higher volume centers did not have significantly lower rates of postoperative complications but were best able to mitigate the adverse effects of these complications on both short- and long-term survival. These data suggest that identifying and implementing the institutional practices that lead to better management of postoperative complications after LTx in high volume centers may be prudent to improving outcomes in lower volume hospitals.

\section{References}

1. Weiss ES, Allen JG, Meguid RA, Patel ND, Merlo CA, Baumgartner WA, et al. The impact of center volume on survival in lung transplantation: an analysis of more than 10,000 cases. Ann Thorac Surg. 2009;88:1062-70.
2. Scarborough JE, Bennett KM, Davis RD, Lin SS, Tracey ET, Kuo PC, et al. Temporal trends in lung transplant center volume and outcomes in the United States. Transplantation. 2010;89:639-43.

3. Birkmeyer JD, Siewers AE, Finlayson EV, Stukel TA, Lucas FL, Batista I, et al. Hospital volume and surgical mortality in the United States. N Engl J Med. 2002; 346:1128-37.

4. Birkmeyer JD, Finlayson EV, Birkmeyer CM. Volume standards for high-risk surgical procedures: potential benefits of the Leapfrog initiative. Surgery. 2001; 130:415-22

5. Hewitt ME, Petitti DB, National Cancer Policy Board (U.S.), National Research Council (U.S.), Division on Earth and Life Studies. Interpreting the VolumeOutcome Relationship in the Context of Cancer Care. Washington (DC): National Academy Press; 2001.

6. Pasquali SK, Li JS, Burstein DS, Sheng S, O’Brien SM, Jacobs ML, et al. Association of center volume with mortality and complications in pediatric heart surgery. Pediatrics. 2012;129:e370-6.

7. Ghaferi AA, Birkmeyer JD, Dimick JB. Hospital volume and failure to rescue with high-risk surgery. Med Care. 2011;49:1076-81.

8. Smith DL, Elting LS, Learn PA, Raut CP, Mansfield PF. Factors influencing the volume-outcome relationship in gastrectomies: a population-based study. Ann Surg Oncol. 2007; 14:1846-52.

9. Aiken LH, Clarke SP, Sloane DM, Sochalski J, Silber JH. Hospital nurse staffing and patient mortality, nurse burnout, and job dissatisfaction. JAMA. 2002;288: 1987-93.

10. Aiken LH, Clarke SP, Cheung RB, Sloane DM, Silber JH. Education levels of hospital nurses and surgical patient mortality. JAMA. 2003;290:1617-23.

11. Low DE, Kunz S, Schembre D, Otero H, Malpass T, Hsi A, et al. Esophagectomy - it's not just about mortality anymore: standardized perioperative clinical pathways improve outcomes in patients with esophageal cancer. J Gastrointest Surg. 2007;11:1395-402.

12. Yeats M, Wedergren S, Fox N, Thompson JS. The use and modification of clinical pathways to achieve specific outcomes in bariatric surgery. Am Surg. 2005;71: $152-4$.

13. DeSomma M, Divekar A, Galloway AC, Colvin SB, Artman M, Auslender M. Impact of a clinical pathway on the postoperative care of children undergoing surgical closure of atrial septal defects. Appl Nurs Res. 2002;15:243-8.

\section{Discussion}

Dr Matthew Bacchetta (New York, NY). I thank the Association for the opportunity to comment on this excellent paper and I thank the authors and Dr Kilic for providing me a copy well in advance.

The theme of superiority of high volume centers versus low volume centers with respect to quality of outcome is certainly not new, yet it continues to expand over a range of operative procedures from the abdomen to the chest. The Johns Hopkins group reported a similar paper in 2009 at the meeting of The Society of Thoracic Surgeons about the value of high volume centers for improved outcome. We have another paper here that suggests similar conclusions, yet there are clearly some major differences in the focus, analysis, observations, and conclusions. In essence, your analysis found that postoperative complication rates were approximately the same across all groups, suggesting that we have standardized our preoperative assessment of patients and standardized our immunosuppression and surgical techniques, with only slight institutional variability, which has led to uniform complication rates across your groups, yet the short-term and long-term impact of these complications was significantly influenced by center volume. Your study suggests that anybody can build a car but only a few know how to fix it when it breaks. Begging the question is, are high volume centers better at fixing cars than low volume centers simply because they have more service centers? They have more staff, transplant pulmonologists, and so on. With that in mind, I have a few technical questions and a few more policyoriented questions. 
The paper from 2009 used 20 cases per year as the inflection point for low and high volume. In your analysis you have come up with 21.8. You chose to use tertiles as opposed to the quartiles that were used in 2009. It seems a bit arbitrary. I hope that you will delve into that a little bit. Is there a meaningful difference between a center that is doing 20 transplants per year and one that's doing 22 ? Why not analyze the outcomes of centers doing 5 or 10 or less per year as ultra-low volume centers? Do you have any data that might hint at that outcome of these ultra-low volume centers?

Is the difference in outcome borne from a deficit in staffing at low volume centers, which can easily become overwhelmed when a major postoperative complication occurs-anastomotic leaks, primary graft dysfunction, renal failure, and so forth? At our institution we have many transplant pulmonologists who are dedicated to this, and it makes it easier to manage these types of complications. Is it really simply a matter of staffing? There are some low volume centers that have very good outcomes, as good as high volume centers. How does your study address the issue of what makes for a center of excellence in lung transplantation?

Can we use your study to implement changes in lung transplantation that will improve national outcomes? Given that $35 \%$ of all the lung transplants during your study period were done by low volume centers, that is a big number and has huge policy implications and staffing implications. The hope of a lot of epidemiologic studies is to provide some guidance for policy recommendations. Should we conclude that lung transplantation should be restricted to centers that do 22 transplants or more, or does this provide the ground for regionalization?

Dr Kilic. Thank you, Dr Bacchetta, for those comments and questions.

We modeled center volume both as a continuous variable, where it was found to have significant impact at each of the time points, as well as a categorical variable. Defining it according to a categorical variable really was done so that we could plot Kaplan-Meier analyses; otherwise, there is really no way to do it when you have it as a continuous variable, and we thought that the best way to do it would be to divide the centers into equal-sized patient tertiles. We did not show the data here, but if you plot annual center volume as a continuous variable and look at mortality on the y-axis, the best discriminatory threshold for survival is around 20, and that is with both uncomplicated and complicated cases. I do think that is the optimal volume threshold. Having said that, though, and this ties in with your question about regionalization, although taken as a whole, the high volume centers have improved outcomes, there is a cohort of low volume centers that consistently have good outcomes every year. I do not think this information is meant to penalize all the low volume centers, but I think it does indicate that we need to identify what the specific practices are in the high volume centers so that we can translate them into the poorly performing low volume centers. In terms of what those practices may be specifically relating to complications, if we talk about identifying complications early, I think it does have to do with staffing. Studies have shown that nurse/patient ratios, for instance, or qualifications of nurses have an important impact on identifying complications and rescue from complication rates. In terms of managing the complications once they are identified, I think availability of consult services, individual surgeon experience, and other center-level factors would have an important impact. Perhaps another important step would be to have UNOS and some of these other registries start to collect institutional variables beyond volume so that we can at least start to study them and see which ones are important factors in contributing to outcomes.

Dr Thomas K. Waddell (Toronto, Ontario, Canada). I want to ask about the statistics of the hazard ratio that you gave for later time periods, 1 and 5 years. I understand the concept of failure to rescue; somebody has a bad complication and you have to see a lot of them in order to rescue them and pull them out of the intensive care unit. I am unclear about the impact of early complications on 5-year mortality as a failure to rescue model. I do not quite understand that. Did you actually model hazard function as 2 functions, an early acute risk and a slower plateau phase kind of hazard? If you did find that the plateau phase hazard was in fact affected by center volume, what do you think is the medical equivalent of failure to rescue in that situation?

Dr Kilic. Thank you for that question. We did actually look at conditional survival as well. When you incorporate conditional survival to 90 days, there is still an important center volume effect beyond that to the 1-year and 5-year mark. I do agree that at 1 year and 5 years, it is hard to delineate exactly how much of that is related to the complication versus other factors that may develop in the longer term. Our analysis does not show the mortalities related to these complications. It simply shows that in patients who have a complication, the survival is better when they are at a higher volume center, and we can presume that it may be related to that complication. Renal failure, for instance, causes a dramatic decline in survival. It is the worst of all the complications. We can presume that it is due to that. However, again, this study was designed for associative rather than causal relationships.

Dr Stephan Schueler (Newcastle upon Tyne, United Kingdom). I come from a high volume lung transplant center. We do about 60 a year. The striking thing in your survival curves was actually the fact that the 30-day drop in survival was identical among all the centers. You would expect that this early attrition rate would be much less in high volume centers, that is, the experienced centers, because of their better judgment. Based on early or bad experience, they should have better judgment in terms of picking the better donor and managing the patients better. My second question is, would your chest physicians now refer patients to high volume centers according to your data?

Dr Kilic. To answer the first question, the effect was actually the strongest in the short term. If you look at the hazard ratios in the risk-adjusted analysis, the hazard ratio is actually the highest at the 90-day mark and then weans down from there into the 1 -year and 5-year marks. The effect is exactly what you are saying. It is actually the greatest in the short term and then reduces in the longer term.

To answer your second question, again, I do not think this information is meant to say that we should regionalize all lung transplants to high volume centers, inasmuch as some low volume centers perform well. I think it just says that we need to do more research to identify exactly why these volume-outcome relationships exist and what we can do to implement those practices in poorly performing low volume centers. 